\title{
A Posteriori Improvements for Interpolating Periodic Splines
}

\author{
By Thomas R. Lucas*
}

\begin{abstract}
A method of a posteriori improvements of interpolating periodic splines of order $2 r$ and their derivatives over a uniform mesh is developed using polynomial-type correction terms. These improvements enhance the order of convergence by several powers of the step size $h$ and are convenient and inexpensive to implement. The polynomials are specified in closed form using the Bernoulli numbers. That the first of these is related to the Bernoulli polynomial of degree $2 r$ is due to Swartz [10], but no general development beyond the first has previously been made. These polynomials are multiplied by high order derivatives of the function evaluated at the mesh points. Some recent results by Lucas [8] are used to accurately estimate these values. Some numerical results are given which correspond closely with the predictions of the theory.
\end{abstract}

1. Introduction. For notation let $C_{p}^{n}[a, b]$ denote the class of all functions $f \in C^{n}(-\infty, \infty)$ such that $f$ is periodic of period $b-a . S$ is said to be a periodic spline of order $2 r$ over a partition of $[a, b], \pi_{n}: a=x_{0}<x_{1}<\cdots<x_{n}=b$, if $S$ restricted to $\left(x_{l-1}, x_{l}\right)$ is a polynomial of degree $2 r-1(1 \leqslant l \leqslant n), S(a)=S(b)$ and $\bar{S} \in C_{p}^{2 r-2}[a, b]$ where $\bar{S}$ is the periodic extension of $S$. If also $S\left(x_{l}\right)=f\left(x_{l}\right)$, $1 \leqslant l \leqslant n$ for $f \in C_{p}^{0}[a, b]$, we say that $S$ is the (smooth) periodic interpolating spline to $f$ of order $2 r$ over the mesh $\pi_{n}$. Throughout this paper the mesh $\pi_{n}$ will be uniform, hence $x_{l}=a+l h, 0 \leqslant l \leqslant n$ where $h=(b-a) / n$. The function $f$ will be in various spaces $C_{p}^{m}[a, b] . S^{(s)}\left(x_{l}\right)$ and $f^{(s)}\left(x_{l}\right)$ will be denoted by $S_{l}^{(s)}$ and $f_{l}^{(s)}$.

It has been known (Ahlberg, Nilson and Walsh [2]) for many years that if $S$ is the periodic interpolating spline of order $2 r$ to $f \in C_{p}^{2 r}[a, b]$, then

$$
f^{(j)}(x)-S^{(j)}(x)=O\left(h^{2 r-j}\right), \quad 0 \leqslant j \leqslant 2 r-1 .
$$

In Section 2 a method is developed for increasing the global accuracy of such periodic spline approximations by several powers of $h$ by adding to $S$ certain polynomial correction terms. It is shown that if $f \in C_{p}^{2 r+4}[a, b]$, then

$$
\begin{aligned}
f^{(j)}\left(x_{l}+\lambda h\right)= & S^{(j)}\left(x_{l}+\lambda h\right)+\sum_{i=0}^{3} \frac{h^{2 r+i-j}}{(2 r+i) !} P_{i}^{(j)}(\lambda) f_{l}^{(2 r+i)} \\
& +O\left(h^{2 r+4-j}\right), \quad 0 \leqslant j \leqslant 2 r, 0 \leqslant \lambda \leqslant 1,
\end{aligned}
$$

Received September 29, 1981; revised June 21, 1982.

1980 Mathematics Subject Classification. Primary 41A15, 41A60, 65D07.

Key words and phrases. Splines, periodic, asymptotic expansion, interpolation, a posteriori corrections, Bernoulli polynomials, Bernoulli numbers.

* This research was supported in part by a grant from the Foundation of the University of North Carolina. 
where $P_{i}$ is a polynomial of degree $2 r+i$ depending only on $r$. Here,

$$
P_{0}(\lambda)=\sum_{i=0}^{2 r-2}\left(\begin{array}{c}
2 r \\
i
\end{array}\right) B_{i} \lambda^{2 r-i}=B_{2 r}(\lambda)-B_{2 r}
$$

where the $B_{i}$ are Bernoulli numbers (see [1], where for reference $B_{0}=1, B_{1}=-1 / 2$, $B_{2}=1 / 6, B_{4}=-1 / 30, B_{6}=1 / 42, B_{8}=-1 / 30$, etc. with $B_{i}=0$ for $i>1$ and odd), and $B_{2 r}(\lambda)$ is the $2 r$ th Bernoulli polynomial [1]. The polynomials $P_{1}, P_{2}$ and $P_{3}$ are also given in closed form in terms of Bernoulli numbers.

Next, a recent result of Lucas [8], that for $f \in C_{p}^{4 r}[a, b]$,

$$
f_{l}^{(2 r)}-\frac{S_{l+1}^{(2 r-2)}-2 S_{l}^{(2 r-2)}+S_{l-1}^{(2 r-2)}}{h^{2}}=O\left(h^{2 r}\right),
$$

will be used to develop practical estimates of $f_{l}^{(2 r)}, f_{l}^{(2 r+1)}$ and $f_{l}^{(2 r+2)}$. Together these results lead to a powerful but computationally simple method of a posteriori corrections to (1.1) which improve the order of convergence by three powers of $h$. These methods could be extended to give even higher orders of improvement.

The polynomials $P_{i}$ will be derived in general closed form using some results previously developed by the author [8] concerning asymptotic expansions of periodic interpolating splines. The main results will be given in Section 2 where (1.2) will be developed using (1.4). Some numerical results will be given in Section 3 which conform very closely with the predictions of the theory.

The first term of the expansion (1.2),

$$
f\left(x_{l}+\lambda h\right)=S\left(x_{l}+\lambda h\right)+h^{2 r} P_{0}(\lambda) f_{l}^{(2 r)} /(2 r) !+O\left(h^{2 r+1}\right),
$$

with the closed form formula (1.3) for $P_{0}$, was found by Swartz [10], [11], but he did not have (1.4) available. Fyfe [4], considering quintic splines only $(r=3)$, found both $P_{0}$ and $P_{1}$ in numerical form using the heuristic methods of Curtis and Powell [3]. He did not develop or apply (1.4) as specialized to quintics. Rosenblatt [9] has recently rediscovered a form of $(1.3)$ as $B_{4}(\lambda)-B_{4}$ for the special case of cubic splines. Innes [6], in working with quintic splines, rediscovered Fyfe's $P_{0}$ and $P_{1}$ and developed for the first time $P_{2}$ and $P_{3}$ in numerical form. Using heuristic methods, he developed (1.4) as specialized to quintics and applied it to estimate the derivatives in (1.2).

While these results have been developed for periodic interpolating splines, it is to be expected that for end conditions of sufficiently high order (for some good choices for cubics, see Lucas [7]; for quintics, see Innes [6]) similar results will hold for (smooth) interpolating splines over a finite interval, and this will lead to some interesting applications of these results.

2. High Order Approximations to $f$ and Its Derivatives. Theorem 1 below uses some recent results by Lucas [8] concerning asymptotic expansions of periodic interpolating splines to display correction terms, in the form of polynomials, which increase the accuracy of the spline approximate by four powers of $h$. A practical and effective way of estimating the terms $f_{l}^{(2 r+k)}$ for low values of $k$ will be presented later. 
THEOREM 1. If $S$ is the periodic interpolating spline of $f$ of order $2 r, r \geqslant 2$, over a uniform mesh of $[a, b]$, then for $f \in C_{p}^{2 r+4}[a, b]$, and $0 \leqslant \lambda \leqslant 1$,

$$
f\left(x_{l}+\lambda h\right)=S\left(x_{l}+\lambda h\right)+\sum_{k=0}^{3} \frac{h^{2 r+k}}{(2 r+k) !} f_{l}^{(2 r+k)} P_{k}(\lambda)+O\left(h^{2 r+4}\right),
$$

where

$$
P_{0}(\lambda)=\sum_{i=0}^{2 r-2}\left(\begin{array}{c}
2 r \\
i
\end{array}\right) B_{i} \lambda^{2 r-i}=B_{2 r}(\lambda)-B_{2 r}
$$

$B_{2 r}(\lambda)$ is the $2 r$ th Bernoulli polynomial and $B_{i}$ is the ith Bernoulli number,

$$
\begin{aligned}
P_{1}(\lambda)= & \sum_{i=0}^{2 r}\left(\begin{array}{c}
2 r+1 \\
i
\end{array}\right) B_{i}(1-i) \lambda^{2 r+1-i}-\left(\begin{array}{c}
2 r+1 \\
1
\end{array}\right) B_{2 r} \lambda^{1} \\
P_{2}(\lambda)= & \sum_{i=0}^{2 r}\left(\begin{array}{c}
2 r+2 \\
i
\end{array}\right) B_{i} \frac{(1-i)(2-i)}{2 !} \lambda^{2 r+2-i}-\left(\begin{array}{c}
2 r+2 \\
2
\end{array}\right) B_{2 r} \lambda^{2} \\
P_{3}(\lambda)= & \sum_{i=0}^{2 r+2}\left(\begin{array}{c}
2 r+3 \\
i
\end{array}\right) B_{i} \frac{(1-i)(2-i)(3-i)}{3 !} \lambda^{2 r+3-i} \\
& -\left(\begin{array}{c}
2 r+3 \\
3
\end{array}\right) B_{2 r} \lambda^{3}-\left(\begin{array}{c}
2 r+3 \\
1
\end{array}\right)\left(\begin{array}{c}
2 r+1 \\
2
\end{array}\right) B_{2 r+2} \lambda^{1}
\end{aligned}
$$

Remark 1. If $f \in C_{p}^{2 r+q}[a, b]$ for $1 \leqslant q \leqslant 3$, then $f\left(x_{l}+\lambda h\right)$ may be approximated by use of the first $q$ correction terms and will be of order $O\left(h^{2 r+q}\right)$.

Proof. Expanding $f$ and $S$ at $\bar{x}=x_{l}+\lambda h$ about $x_{l}$ gives

$$
f(\bar{x})-S(\bar{x})=\sum_{j=1}^{2 r-1} \frac{\lambda^{j} h^{j}}{j !}\left(f_{l}^{(j)}-S_{l}^{(j)}\right)+\sum_{j=2 r}^{2 r+3} \frac{\lambda^{j} h^{j}}{j !} f_{l}^{(j)}+O\left(h^{2 r+4}\right) .
$$

By Theorem 1 and Remark 1 of Lucas [8]

$$
\begin{aligned}
f_{l}^{(j)}-S_{l}^{(j)}= & -\sum_{k=0}^{1} A_{2 k, r}^{(j)} h^{2 r+2 k-\hat{j}} f_{l}^{(2 r+2 k+j-\hat{j})} \\
& +O\left(h^{2 r+4-j}\right), \quad 1 \leqslant j \leqslant 2 r-2,
\end{aligned}
$$

where

$$
\begin{aligned}
A_{2 k, r}^{(j)}= & \frac{1}{(2 r-1) !}\left[\frac{(2 k+j-\hat{j}) !}{(2 k-\hat{j}) !}-(-1)^{j} \frac{(2 r-1) !}{(2 r-1-j) !}\right] \\
& \times \frac{B_{2 r+2 k-\hat{j}}}{(2 k+j-\hat{j}) !(2 r+2 k-\hat{j})},
\end{aligned}
$$

and $\hat{j}$ equals $j$ for $j$ even and $j-1$ for $j$ odd. By convention the term

$$
(2 k+j-\hat{j}) ! /(2 k-\hat{j}) !
$$

is taken to be zero if $\hat{j}>2 k$. From Lucas [8, Theorem 2],

$$
\begin{aligned}
f_{l}^{(2 r-1)}-S_{l^{+}}^{(2 r-1)}= & -\frac{h}{2} f_{l}^{(2 r)}-\frac{B_{2}}{2 !} h^{2} f_{l}^{(2 r+1)} \\
& -\frac{B_{4}}{4 !}\left(1+\delta_{r 2}\right) h^{4} f_{l}^{(2 r+3)}+O\left(h^{5}\right),
\end{aligned}
$$


where $\delta_{r k}$ is the Kronecker delta. Substituting (2.7) and (2.9) into (2.6), and noting that $B_{1}=-\frac{1}{2}$, gives

$$
\begin{aligned}
f(\bar{x})-S(\bar{x})= & \sum_{j=1}^{2 r-2} \frac{\lambda^{j}}{j !}\left(-\sum_{k=0}^{1} A_{2 k, r}^{(j)} h^{2 r+2 k+j-j} f_{l}^{(2 r+2 k+j-j)}\right) \\
& -\frac{\lambda^{2 r-1}}{(2 r-1) !}\left(-B_{1} h^{2 r} f_{l}^{(2 r)}+\frac{B_{2}}{2 !} h^{2 r+1} f_{l}^{(2 r+1)}\right. \\
& \left.+\frac{B_{4}}{4 !}\left(1+\delta_{r 2}\right) h^{2 r+3} f_{l}^{(2 r+3)}\right) \\
& +\sum_{j=2 r}^{2 r+3} \frac{\lambda^{j} h^{j}}{j !} f_{l}^{(j)}+O\left(h^{2 r+4}\right) .
\end{aligned}
$$

This can be rewritten considering the cases $k=0$ or $1 ; j$ even or odd as:

$$
\begin{aligned}
f(\bar{x})-S(\bar{x})= & \left.-\sum_{j=1}^{r-1} \frac{A_{0, r}^{(2 j)}}{(2 j) !} \lambda^{2 j}+\frac{B_{1}}{(2 r-1) !} \lambda^{2 r-1}+\frac{B_{0}}{(2 r) !} \lambda^{2 r}\right) h^{2 r} f_{l}^{(2 r)} \\
& +\left(-\sum_{j=0}^{r-2} \frac{A_{0, r}^{(2 j+1)}}{(2 j+1) !} \lambda^{2 j+1}-\frac{B_{2}}{(2 r-1) !} \frac{\lambda^{2 r-1}}{2 !}\right. \\
& \left.+\left(-\sum_{j=1}^{r-1} \frac{A_{2, r}^{(2 j)}}{(2 j) !} \lambda^{2 j}+\frac{B_{0}}{(2 r+2) !} \lambda^{2 r+2}\right) h^{2 r+2} f_{l}^{(2 r+2)}\right) h^{2 r+1} f_{l}^{(2 r+1)} \\
& +\left(-\sum_{j=0}^{r-2} \frac{A_{2, r}^{(2 j+1)}}{(2 j+1) !} \lambda^{2 j+1}-\frac{\left(1+\delta_{r 2}\right)}{(2 r-1) !} \frac{B_{4}}{4 !} \lambda^{2 r-1}\right. \\
& \left.+O\left(h^{2 r+4}\right) \quad+\frac{B_{0}}{(2 r+3) !} \lambda^{2 r+3}\right) h^{2 r+3} f_{l}^{(2 r+3)} \\
= & \sum_{k=0}^{3} \frac{h^{2 r+k}}{(2 r+k) !} f_{l}^{(2 r+k)} P_{k}(\lambda)+O\left(h^{2 r+4}\right)
\end{aligned}
$$

Using (2.8) to evaluate $A_{2 k, r}^{(j)}$ for $k=0$ and 1 gives

(2.11a) $\quad A_{0, r}^{(2 j)}=-\frac{B_{2 r-2 j}}{(2 r-2 j) !}$,

(2.11b) $A_{0, r}^{(2 j+1)}=\frac{B_{2 r-2 j}}{(2 r-1) !(-2 j) !(2 r-2 j)}+\frac{B_{2 r-2 j}}{(2 r-2 j) !}(2 r-1-2 j)$,

(2.11c) $\quad A_{2, r}^{(2 j)}=\frac{B_{2 r+2-2 j}}{(2 r-1) !(2-2 j) !(2 r+2-2 j)}$

$$
-\frac{B_{2 r+2-2 j}}{(2 r+2-2 j) ! 2 !}(2 r-2 j)(2 r+1-2 j) \text {, }
$$


(2.11d)

$$
\begin{aligned}
A_{2, r}^{(2 j+1)}= & \frac{B_{2 r+2-2 j}}{(2 r-1) !(2-2 j) !(2 r+2-2 j)} \\
& +\frac{B_{2 r+2-2 j}}{(2 r+2-2 j) ! 3 !}(2 r-1-2 j)(2 r-2 j)(2 r+1-2 j) .
\end{aligned}
$$

Applying (2.11) to the polynomials $P_{k}(\lambda)$ defined by (2.10), and recalling the convention on negative factorials, will lead to (2.2)-(2.5):

$$
\begin{aligned}
& P_{0}(\lambda)=\sum_{j=1}^{r-1}\left(\begin{array}{c}
2 r \\
2 j
\end{array}\right) B_{2 r-2 j} \lambda^{2 j}+\left(\begin{array}{c}
2 r \\
1
\end{array}\right) B_{1} \lambda^{2 r-1}+\left(\begin{array}{c}
2 r \\
0
\end{array}\right) B_{0} \lambda^{2 r} \\
& =\sum_{i=0}^{2 r-2}\left(\begin{array}{c}
2 r \\
i
\end{array}\right) B_{i} \lambda^{2 r-i}=B_{2 r}(\lambda)-B_{2 r} \\
& P_{1}(\lambda)=-\left(\begin{array}{c}
2 r+1 \\
1
\end{array}\right) B_{2 r} \lambda^{1}-\sum_{j=0}^{r-2}\left(\begin{array}{c}
2 r+1 \\
2 j+1
\end{array}\right) B_{2 r-2 j}(2 r-1-2 j) \lambda^{2 j+1} \\
& -\left(\begin{array}{c}
2 r+1 \\
2
\end{array}\right) B_{2} \lambda^{2 r-1}+\left(\begin{array}{c}
2 r+1 \\
0
\end{array}\right) B_{0} \lambda^{2 r+1} \\
& =\sum_{i=0}^{2 r}\left(\begin{array}{c}
2 r+1 \\
i
\end{array}\right) B_{i}(1-i) \lambda^{2 r+1-i}-\left(\begin{array}{c}
2 r+1 \\
1
\end{array}\right) B_{2 r} \lambda^{1} \\
& P_{2}(\lambda)=-\left(\begin{array}{c}
2 r+2 \\
2
\end{array}\right) B_{2 r} \lambda^{2}+\sum_{j=1}^{r-1}\left(\begin{array}{c}
2 r+2 \\
2 j
\end{array}\right) B_{2 r+2-2 j} \frac{(2 r-2 j)(2 r+1-2 j)}{2 !} \lambda^{2 j} \\
& +\left(\begin{array}{c}
2 r+2 \\
0
\end{array}\right) B_{0} \lambda^{2 r+2} \\
& =\sum_{i=0}^{2 r}\left(\begin{array}{c}
2 r+2 \\
i
\end{array}\right) B_{i} \frac{(1-i)(2-i)}{2 !} \lambda^{2 r+2-i}-\left(\begin{array}{c}
2 r+2 \\
2
\end{array}\right) B_{2 r} \lambda^{2}, \\
& P_{3}(\lambda)=-\frac{(2 r+3) !}{(2 r-1) ! 2 !(2 r+2)} B_{2 r+2} \lambda^{1}-\frac{(2 r+3) !}{(2 r-1) ! 3 ! 2 r} B_{2 r} \lambda^{3}\left(1-\delta_{r 2}\right) \\
& -\sum_{j=0}^{r-2}\left(\begin{array}{l}
2 r+3 \\
2 j+1
\end{array}\right) B_{2 r+2-2 j} \frac{(2 r-1-2 j)(2 r-2 j)(2 r+1-2 j)}{3 !} \lambda^{2 j+1} \\
& -\left(\frac{2 r+3}{4}\right) B_{4}\left(1+\delta_{r 2}\right) \lambda^{2 r-1}+\left(\begin{array}{c}
2 r+3 \\
0
\end{array}\right) B_{0} \lambda^{2 r+3} \\
& =\sum_{i=0}^{2 r+2}\left(\begin{array}{c}
2 r+3 \\
i
\end{array}\right) B_{i} \frac{(1-i)(2-i)(3-i)}{3 !} \lambda^{2 r+3-i}-\left(\begin{array}{c}
2 r+3 \\
3
\end{array}\right) B_{2 r} \lambda^{3} \\
& -\left(\begin{array}{c}
2 r+3 \\
1
\end{array}\right)\left(\begin{array}{c}
2 r+1 \\
2
\end{array}\right) B_{2 r+2} \lambda^{1} \\
& +\left[\left(\begin{array}{c}
2 r+3 \\
3
\end{array}\right) B_{2 r} \lambda^{3}-\left(\begin{array}{c}
2 r+3 \\
4
\end{array}\right) B_{4} \lambda^{2 r-1}\right] \delta_{r 2} .
\end{aligned}
$$

The last term in $P_{3}$ is zero for all $r$. 
Remark 2. For cubic $(r=2)$ and quintic $(r=3)$ splines, the polynomials of Theorem 1 are:

for cubic splines

$$
\begin{aligned}
& P_{0}(\lambda)=\lambda^{4}-2 \lambda^{3}+\lambda^{2} \\
& P_{1}(\lambda)=\lambda^{5}-\frac{5}{3} \lambda^{3}+\frac{2}{3} \lambda \\
& P_{2}(\lambda)=\lambda^{6}-\lambda^{2} \\
& P_{3}(\lambda)=\lambda^{7}+\frac{7}{3} \lambda^{3}-\frac{10}{3} \lambda
\end{aligned}
$$

for quintic splines

$$
\begin{aligned}
& P_{0}(\lambda)=\lambda^{6}-3 \lambda^{5}+\frac{5}{2} \lambda^{4}-\frac{1}{2} \lambda^{2}, \\
& P_{1}(\lambda)=\lambda^{7}-\frac{7}{2} \lambda^{5}+\frac{7}{2} \lambda^{3}-\lambda, \\
& P_{2}(\lambda)=\lambda^{8}-7 \lambda^{4}+6 \lambda^{2}, \\
& P_{3}(\lambda)=\lambda^{9}+\frac{21}{5} \lambda^{5}-22 \lambda^{3}+\frac{84}{5} \lambda
\end{aligned}
$$

THEOREM 2. Under the hypotheses of Theorem 1 , for $0 \leqslant \lambda \leqslant 1$ and $0 \leqslant j \leqslant 2 r$,

$$
\begin{aligned}
f^{(j)}\left(x_{l}+\lambda h\right)= & S^{(j)}\left(x_{l}+\lambda h\right)+\sum_{k=0}^{3} \frac{h^{2 r+k-j}}{(2 r+k) !} f_{l}^{(2 r+k)} P_{k}^{(j)}(\lambda) \\
& +O\left(h^{2 r+4-j}\right) .
\end{aligned}
$$

Proof. We will first consider the case $j=2 r$. By Taylor's Theorem

$$
f^{(2 r)}\left(x_{l}+\lambda h\right)=\sum_{k=0}^{3} \frac{(\lambda h)^{k}}{k !} f_{l}^{(2 r+k)}+O\left(h^{4}\right),
$$

while a direct calculation from (2.2)-(2.5) gives

$$
P_{k}^{(2 r)}(\lambda)=(2 r+k) ! \frac{\lambda^{k}}{k !}, \quad 0 \leqslant k \leqslant 3 .
$$

Solving (2.14) for $\lambda^{k} / k$ ! and substituting into (2.13) gives

$$
f^{(2 r)}\left(x_{l}+\lambda h\right)=\sum_{k=0}^{3} \frac{h^{k}}{(2 r+k) !} f_{l}^{(2 r+k)} P_{k}^{(2 r)}(\lambda)+O\left(h^{4}\right),
$$

which is $(2.12)$ for $j=2 r$. The result now follows from (2.15) by induction using successive definite integrations: suppose for some $j, 0<j \leqslant 2 r$,

$$
\begin{aligned}
f^{(j)}\left(x_{l}+\lambda h\right)= & S^{(j)}\left(x_{l}+\lambda h\right)+\sum_{k=0}^{3} \frac{h^{2 r-j+k}}{(2 r+k) !} f_{l}^{(2 r+k)} P_{k}^{(j)}(\lambda) \\
& +O\left(h^{2 r+4-j}\right)
\end{aligned}
$$

Integrating with respect to $\lambda$ from 0 to $\lambda$ gives $(2.16)$ for $j-1$ provided that

$$
f^{(s)}\left(x_{l}\right)=S^{(s)}\left(x_{l}\right)+\sum_{k=0}^{3} \frac{h^{2 r-s+k}}{(2 r+k) !} f_{l}^{(2 r+k)} P_{k}^{(s)}(0)+O\left(h^{2 r+4-s}\right)
$$


with $s=j-1$. But from (2.10)

$$
\begin{aligned}
& P_{0}^{(2 r-1)}(0)=(2 r) ! B_{1}, \\
& P_{k}^{(2 r-1)}(0)=\frac{-(2 r+k) !}{(k+1) !}\left(1+\delta_{r, k-1}\right) B_{k+1}, \quad 1 \leqslant k \leqslant 3,
\end{aligned}
$$

which validates (2.17) for $s=2 r-1$ by (2.9). From (2.10), for $0 \leqslant s \leqslant 2 r-2$, $k=0$ or 2 ,

$$
P_{k}^{(s)}(0)= \begin{cases}-A_{k, r}^{(s)}(2 r+k) !, & s \text { even } \\ 0, & s \text { odd }\end{cases}
$$

For $k=1$ or 3 ,

$$
P_{k}^{(s)}(0)= \begin{cases}0, & s \text { even }, \\ -A_{k-1, r}^{(s)}(2 r+k) !, & s \text { odd } .\end{cases}
$$

So (2.17) is established by comparison with (2.7).

Corollary 1. Under the hypotheses of Theorem 2, for $f \in C_{p}^{(2 r+1)}[a, b]$, and $s$ odd, $1 \leqslant s \leqslant 2 r-1$,

$$
f^{(s)}\left(x_{l}+.5 h\right)=S^{(s)}\left(x_{l}+.5 h\right)+O\left(h^{2 r+1-s}\right) .
$$

Proof. $P_{0}^{(s)}(.5)=B_{2 r}^{(s)}(.5)=0$, for odd $s, 1 \leqslant s \leqslant 2 r-1$.

Note. This result was first proven by Swartz [10].

Theorem 2 is an extremely practical result as $f^{(2 r)}\left(x_{l}\right)$ can be very accurately estimated by use of (1.4), and $f^{(2 r+i)}\left(x_{l}\right)$ can be estimated from these results for low values of $i$ :

THEOREM 3. If $S$ is the periodic interpolating spline of order $2 r$ of $f \in C_{p}^{2 r+3}[a, b]$, then

$$
\begin{aligned}
f_{l}^{(2 r)} & =\frac{S_{l+1}^{(2 r-2)}-2 S_{l}^{(2 r-2)}+S_{l-1}^{(2 r-2)}}{h^{2}}+O\left(h^{3}\right), \\
f_{l}^{(2 r+1)} & =\frac{S_{l+2}^{(2 r-2)}-2 S_{l+1}^{(2 r-2)}+2 S_{l-1}^{(2 r-2)}-S_{l-2}^{(2 r-2)}}{2 h^{3}}+O\left(h^{2}\right), \\
f_{l}^{(2 r+2)} & =\frac{\delta^{4} S_{l}^{(2 r-2)}}{h^{4}}+O(h) .
\end{aligned}
$$

Proof. Equation (2.18) is a consequence of Theorem 3 and Remark 5 of Lucas [8]. Equations (2.19)-(2.20) follow from (2.18).

THEOREM 4. If $S$ is the periodic interpolating spline of order $2 r$ of $f \in C_{p}^{2 r+3}[a, b]$, then for $0 \leqslant j \leqslant 2 r$,

$$
\begin{aligned}
f^{(j)}\left(x_{l}+\lambda h\right)= & S^{(j)}\left(x_{l}+\lambda h\right) \\
& +\frac{h^{2 r-j-2}}{(2 r) !}\left(\delta^{2} S_{l}^{(2 r-2)} P_{0}^{(j)}(\lambda)+\frac{\left(\delta^{2} S_{l+1}^{(2 r-2)}-\delta^{2} S_{l-1}^{(2 r-2)}\right)}{2(2 r+1)} P_{1}^{(j)}(\lambda)\right. \\
& \left.+\frac{\delta^{4} S_{l}^{(2 r-2)}}{(2 r+1)(2 r+2)} P_{2}^{(j)}(\lambda)\right) \\
& +O\left(h^{2 r+3-j}\right) .
\end{aligned}
$$


Proof. This is a direct consequence of Theorems 2 and 3.

Remark 3. If only the first (first two) of the above three correction terms is used the error will be $O\left(h^{2 r+1-j}\right)\left(O\left(h^{2 r+2-j}\right)\right)$. In this case $f$ need only be in $C_{p}^{2 r+1}[a, b]$ $\left(C_{p}^{2 r+2}[a, b]\right)$.

3. A Numerical Example. Consider the smooth periodic cubic $(r=2)$ spline interpolant $S$ of $f \in C_{p}^{7}[a, b]$ with uniform mesh $\pi_{n} . S$ may be expressed as

$$
S(x)=\sum_{i=-1}^{n+1} c_{i} \hat{S}\left(\left(x-x_{i}\right) / h\right)
$$

where $\hat{S}$ is the standard $B$-spline basis function for smooth cubic splines with support in $[-2,2]$. Since $\hat{S}(-1)=\hat{S}(1)=1 / 6$ and $\hat{S}(0)=4 / 6$, the interpolation condition leads to the circulant system

$$
c_{i-1}+4 c_{i}+c_{i+1}=6 f\left(x_{i}\right), \quad 0 \leqslant i \leqslant n-1,
$$

which, along with the periodic end conditions $c_{-1}=c_{n-1}$ and $c_{0}=c_{n}$, leads to a symmetric, strictly diagonally dominant circulant matrix equation in $\left\{c_{i}\right\}$. (In (3.1), $c_{n+1}=c_{1}$.) This sparse system may be conveniently and rapidly solved using the Cholesky method with storage required for just the diagonal, subdiagonal, bottom row and right-hand side. For purposes of repeated evaluation of $S$ and its derivatives the results may be reexpressed in terms of the polynomial pieces $\left.S\right|_{\left[x_{i}, x_{i+1}\right]}$ for $0 \leqslant i \leqslant n-1$. In any case, from (3.1)

$$
S_{l}^{(2)}=\left(c_{l-1}-2 c_{l}+c_{l+1}\right) / h^{2}, \quad 0 \leqslant l \leqslant n
$$

as $\hat{S}^{(2)}(-1)=\hat{S}^{(2)}(1)=1$ and $\hat{S}^{(2)}(0)=-2$. An alternate method of computing $S$ is given in Golomb [5].

Thus the expressions $\delta^{2} S_{l}^{(2)}, \delta^{2} S_{l+1}^{(2)}-\delta^{2} S_{l-1}^{(2)}$ and $\delta^{4} S_{l}^{(2)}$ required for the application of Theorem 4 may be easily evaluated and stored in arrays. If the polynomials $P_{i}^{(j)}$ are only to be evaluated for a small set of $\lambda$ 's, this could also be done before use of a general evaluative routine. Alternatively the piecewise cubic polynomial used to approximate $f$ may be replaced with a piecewise polynomial of degree 4,5 or 6 according as to how many levels of correction are desired, with a similar procedure for estimating derivatives of $f$.

Table 1 below illustrates the quality of these results by considering the function $f(x)=\sin x$ over the interval $[0,2 \pi]$ using respectively $0,1,2$ and 3 levels of correction and $n=20$. The observed error rates for $f, f^{\prime}$ and $f^{\prime \prime}$ were experimentally computed, sampling the error at intervals of $h / 12$ and comparing with the observed errors for $n=10$. These observed rates are given in parentheses after the observed errors with $n=20$ for $f, f^{\prime}$ and $f^{\prime \prime}$. A comparison with Theorem 4 and the remark following it shows excellent agreement with the asymptotic rates. 


\section{TABLE 1}

Maximum errors and asymptotic rates for periodic

cubic splines interpolating $\sin x$

\begin{tabular}{c|c|c|c|c}
\hline & $\begin{array}{c}\text { periodic cubic } \\
\text { spline (no corr.) }\end{array}$ & $\begin{array}{c}\text { one corr. } \\
\text { term }\end{array}$ & $\begin{array}{c}\text { two corr. } \\
\text { terms }\end{array}$ & $\begin{array}{c}\text { three corr. } \\
\text { terms }\end{array}$ \\
\hline$f$ & $2.57 \mathrm{E}-5(4.1)$ & $4.40 \mathrm{E}-6(5.1)$ & $5.16 \mathrm{E}-7(6.1)$ & $1.48 \mathrm{E}-7(7.0)$ \\
\hline$f^{\prime}$ & $2.44 \mathrm{E}-4(3.1)$ & $5.48 \mathrm{E}-5(4.1)$ & $8.67 \mathrm{E}-6(5.0)$ & $1.53 \mathrm{E}-6(6.0)$ \\
\hline$f^{\prime \prime}$ & $8.25 \mathrm{E}-3(1.9)$ & $1.59 \mathrm{E}-3(2.9)$ & $2.58 \mathrm{E}-4(3.9)$ & $1.57 \mathrm{E}-5(5.0)$ \\
\hline
\end{tabular}

4. Conclusions. A method of a posteriori corrections has been developed for (smooth) periodic interpolating splines over a uniform mesh. After the interpolating periodic spline has been computed, a few simple additional calculations allow for an improvement by a factor of $h^{3}$ in the error of both the spline and its derivatives at all points.

Department of Mathematics

The University of North Carolina at Charlotte

Charlotte, North Carolina 28223

1. M. Abramowitz \& I. A. Stegun, Handbook of Mathematical Functions, Nat. Bur. Standards Appl. Math. Ser. No. 55, Washington, D. C., 1964.

2. J. H. Ahlberg, E. N. Nilson \& J. L. Walsh, The Theory of Splines and their Applications, Academic Press, New York, 1967.

3. A. R. CuRTis \& M. J. D. Powell, Using Cubic Splines to Approximate Functions of One Variable to Prescribed Accuracy, Atomic Energy Research Establishment, R5602, Harwell, England, 1967.

4. D. J. FyFE, "Linear dependence relations connecting equal interval $N$ th degree splines and their derivatives," J. Inst. Math. Appl., v. 7, 1971, pp. 398-406.

5. M. GolomB, "Approximation by periodic spline interpolants on uniform meshes," J. Approx. Theory, v. 1, 1968, pp. 26-65.

6. N. F. INNES, High Order End Conditions and Convergence Results for Uniformly Spaced Quintic Splines, Research Report 1, Dept. of Mathematics, University of North Carolina at Charlotte, 1979.

7. T. R. LUCAS, "Error bounds for interpolating cubic splines under various end conditions," SIAM J. Numer. Anal., v. 11, 1974, pp. 569-584.

8. T. R. LUCAS, “Asymptotic expansions for interpolating periodic splines," SIAM J. Numer. Anal., v. 19, 1982, pp. 1051-1066.

9. M. Rosenblatt, "Asymptotics and representation of cubic splines," J. Approx. Theory, v. 17, 1976, pp. 332-343.

10. B. SWARTz, “ $O\left(h^{2 n+2-l}\right)$ bounds on some spline interpolation errors," Bull. Amer. Math. Soc., v. 74, 1968, pp. 1072-1078.

11. B. SWARTZ, $O\left(h^{2 n+2-l}\right)$ Bounds on Some Spline Interpolation Errors, Los Alamos Scientific Laboratory LA-3886, 1968. 